\title{
El derecho a la educación y los contenidos prescritos en los curricula diferenciados de Educación Secundaria Obligatoria
}

\author{
Juan García-Rubio. Universidad de Valencia \\ Recepción: 1 de octubre de 2016 | Revisión: 16 de octubre de 2016 | Aceptado: 31 octubre de 2016 \\ Correspondencia: Juan García Rubio | Email: Juan.Garcia-Rubio@uv.es \\ Citar: Garcia-Rubio, J. (2016). El derecho a la educación y los contenidos prescritos en los curricula diferenciados de Educación \\ Secundaria Obligatoria. ReiDoCrea, 5, 288-296.
}

\begin{abstract}
Resumen: Los contenidos del curriculum prescrito deben ser garantía del derecho a la educación que tiene que disfrutar todo el alumnado independientemente de que esté en el aula ordinaria o en un programa extraordinario de atención a la diversidad. Método: Comparación exhaustiva del curriculum oficial o prescrito de la Comunidad Valenciana de $4^{\circ}$ de ESO, $2^{\circ}$ de PDC y $2^{\circ}$ de PCPI en las materias de matemáticas y lengua castellana. Se utiliza el método Delphi para que los expertos elaboren un curriculum básico de ambas materias decidiendo consensuadamente $-80 \%$ de coincidencia- lo que es fundamental y lo que es menos relevante. Resultados: Se muestran grandes diferencias entre los contenidos del curriculum de $4^{\circ}$ de ESO y los que se establecen para ambos programas de atención a la diversidad. Conclusiones: Los contenidos del curriculum prescrito de los curricula diferenciados no garantizan el derecho a la educación del alumnado que asiste a los programas especiales que conducen a la obtención del graduado en Educación Secundaria Obligatoria.
\end{abstract}

Palabras Clave: Plan de estudios | Derecho a la Educación

The right to education and the mandated contents in differentiated curricula of Compulsory Secondary Education

Abstract: The contents of the mandated curriculum must be guaranteed by the right to education, which has to be enjoyed by all students regardless of who is in regular classes or in an extraordinary program of attention to diversity. Method: Complete comparison of the official or mandated curriculum of Valencia Region in 4th ESO, 2nd PDC and 2nd PCPI in Maths and Spanish. The Delphi method is used for experts to develop a core curriculum of both subjects consensually $-80 \%$ coincidence- deciding what is essential and what is less relevant. Results: Large differences between the content of the curriculum of 4th ESO and those established for both programs with attention to diversity. Conclusions: The contents of mandated curriculum of differentiated curriculums do not guarantee the right to education of students attending special programs leading to obtaining the graduate in Compulsory Secondary Education.

Keywords: Curriculum | Right to Education

\section{Introducción}

No debemos equiparar derecho a la educación con el simple acceso del alumnado a la escuela. Para garantizar el derecho a la educación a todos los alumnos y alumnas que acuden a los centros educativos en las etapas obligatorias, éstos tienen que adquirir unos aprendizajes fundamentales y básicos para la vida (Tomasevski, 2004; Torres 2006). No se puede lograr la igualdad absoluta de resultados en la escuela, cada alumno tiene que lograr la mayor de sus potencialidades, pero sí tenemos que aspirar a conseguir que todos alcancen lo que nadie debe ignorar (Bolívar, 2008).

La Administración establece un curriculum oficial o prescrito que sirve de guía a a toda la comunidad educativa del país. Es el punto inicial y de referencia del resto de curricula resultantes en las diferentes fases del desarrollo curricular, esto es, del curriculum presentado a los profesores, del curriculum moldeado por los mismos, del curriculum en acción, del curriculum realizado y del curriculum evaluado (Gimeno, 1989). Sin duda, tiene una gran influencia sobre lo que sucede en la práctica en el aula: en concreto, en los contenidos que explican los profesores a los alumnos, en el orden en que lo hacen, y en los libros de texto utilizados por ambos. El curriculum prescrito es el corazón del sistema educativo de un país, donde se establecen las normas de juego a las que se tienen que adaptar todos los componentes del mismo. Muchos otros factores condicionan la práctica en el aula pero éste es decisivo. No son los profesores, al menos en primera instancia, los que fijan los contenidos a impartir en 
sus aulas sino unos actores muy alejados de las mismas: la Administración. Pero inevitablemente hay que tener un documento que satisfaga el desarrollo de las diferentes funciones de la educación. En palabras de Gimeno (1998: 190), "De algún texto en educación hay que partir porque sin él sería otra cosa: cuidado del menor, terapia, consejo, intercambio personal". En educación, la transmisión de la cultura es un fin fundamental y el curriculum prescrito determina que elementos de la misma han sido seleccionados.

Los componentes del curriculum han ido variando a lo largo del tiempo dependiendo de las distintas leyes educativas que se han ido aprobando en España, pero entre ellos siempre se han encontrado los contenidos. Habrá contenidos que hay que saber (conceptuales), habrá contenidos que hay que saber hacer (procedimentales) y habrá contenidos que comportan saber ser (actitudinales). No obstante, cuando aprendemos cualquier cosa, ésta habitualmente tiene componentes conceptuales, procedimentales y actitudinales, siempre que no sea algo puramente mecánico. No es posible aprender de manera significativa los contenidos procedimentales desligados de los conceptuales y actitudinales (Pozo, 2010).

Por su parte, Gimeno (2014) sostiene que si hay una cuestión que debería ser fundamental en cualquier política educativa, es la de la selección de la cultura escolar, la determinación de cuáles son los contenidos que deben ser enseñados en las clases: esto es, establecer qué contenidos son indispensables y cuáles deben ser relegados, y en función de qué criterios. En esa misma línea, Carbonell señala que los contenidos deberían ser elegidos en función de su "relevancia, es decir, la adquisición de conocimientos básicos con valor cultural y social que nos ayudan a comprender la evolución multidimensional de la humanidad y a comprender el mundo en que vivimos." (Carbonell, 2014: 44).

En la última de las etapas de la escuela obligatoria en España, en la Educación Secundaria Obligatoria, se encuentra alumnado con muy variados intereses, capacidades y rendimientos. Ante esta heterogeneidad de alumnos y alumnas se puede responder de dos maneras muy distintas: una es convirtiéndola en homogeneidad a través de la separación de los alumnos según su nivel, ofreciendo curricula diferenciados. Sin embargo, hay otra alternativa más ambiciosa, que conlleva mayor dificultad para el docente y que es la opuesta a la anterior. Se trata de que, frente a la heterogeneidad y diversidad del alumnado, tengamos como referente un curriculum común para todos. Éste estará dirigido a intentar que los aprendizajes sean, si no iguales para todos, sí lo más parecidos posibles o, por lo menos, similares culturalmente. Desde esta postura, se apuesta por una metodología variada que dé a cada alumno un camino posible para lograr, al menos, unos conocimientos básicos.

El legislador español, desde la Ley Orgánica General del Sistema Educativo -LOGSEde 1990, ha ido estableciendo diferentes programas extraordinarios de atención a la diversidad: Programa de Garantía Social -PGS-, Programa de Diversificación Curricular -PDC-, Programa de Cualificación Profesional Inicial -PCPI-, Programa de Mejora del Aprendizaje y Rendimiento -PMAR-. Estos programas han tenido curricula diferentes al del aula ordinaria. La investigación que vamos a mostrar a continuación se centra en los contenidos de los curricula de los programas establecidos en la Ley Orgánica de Educación -LOE- de 2006, en concreto en el PDC y en el PCPI y el interrogante principal que hemos tratado de responder es el siguiente: ¿Garantiza el legislador el derecho a la educación al alumnado a través de los contenidos que se establecen en estos dos programas extraordinarios? Siguiendo los razonamientos que realiza el profesor Escudero $(2006,2009)$, un determinado programa educativo será de inclusión educativa, garantizará el derecho a la educación, si el legislador persigue que 
los alumnos consigan en estos programas conocimientos parecidos a los del aula ordinaria, utilizando medios y metodología distintos, pero sin cambiar en gran medida otros componentes del curriculum, como los contenidos. Y será de exclusión educativa si lo que la normativa prescribe es una rebaja sustancial en los aprendizajes, de tal manera que un alumno que no obtendría el título de Graduado en ESO en el aula ordinaria podría conseguirlo en un PDC o en un PCPI debido solamente a ese motivo.

\section{Método}

\section{Participantes}

Diez expertos en cada una de las dos materias escogidas en la investigación: matemáticas y lengua castellana. En concreto, en matemáticas participaron dos profesores de cada uno de los programas objeto de la investigación -ESO, PDC y PCPI-, un autor de libros de texto de matemáticas de ESO y tres profesores de universidad especialistas en la materia. En uno de los casos se compartía un doble perfil: el autor de libros de texto también era profesor de ESO. Y en castellano, colaboraron dos profesores de cada uno de los programas, dos profesores de universidad especialistas en esta materia-, un asesor del CEFIRE que también había colaborado en la confección de uno de los dos programas en la Comunidad Valenciana, y un autor de libros de texto.

\section{Instrumentos}

Para contestar a la pregunta formulada, como cada Comunidad Autónoma establece curricula diferentes entre sí, hemos limitado la investigación a la Comunidad Valenciana, al estudio comparativo entre los contenidos establecidos en los curricula que conducían a la obtención del graduado en ESO: $4^{\circ} \mathrm{ESO}, 2^{\circ} \mathrm{PDC}$ y $2^{\circ} \mathrm{PCPI}$. También, a fin de evitar una excesiva extensión del estudio hemos elegido las dos materias del curriculum antes reseñadas. Un aspecto fundamental en la investigación ha sido la cuestión de la objetividad, y para asegurarla hemos recurrido al método Delphi, a la puesta en común de varios expertos, basándonos en la creencia de que "varias subjetividades coincidentes alcanzan un grado de objetividad aceptable, desde un punto de vista profesional" (Casanova, 1995: 117). A esta manera de atenuar la subjetividad de cualquier juicio se le denomina intersubjetividad compartida. El método Delphi, es un proceso sistemático encaminado hacia la obtención, si es posible, de un consenso sobre un tema específico entre un grupo de especialistas en una materia.

\section{Procedimiento}

A los expertos se les presentaban todos los posibles contenidos de los curricula objeto de comparación $-4^{\circ}$ ESO, $2^{\circ}$ PDC y $2^{\circ} \mathrm{PCPI}$-, más los que se establecían en el decreto de Mínimos fijado por el Estado para $4^{\circ}$ de ESO, no repitiendo ninguno que fuese idéntico o muy similar. Los expertos tenían que decidir si cada uno de los contenidos que se les presentaban de la materia era básico o no básico, entendiendo por básico aquello que el alumno no puede ignorar, lo que es esencial para desarrollarse personal y profesionalmente en el futuro y para poder participar en la vida social. Hemos establecido un criterio de consenso, fijado en un número que considerábamos razonablemente alto, éste fue el $80 \%$ de respuestas idénticas (Landeta, 2002).

Hemos tomado como referencia el curriculum de $4^{\circ}$ de ESO determinado por el legislador valenciano para comprobar si lo que se busca con estos dos programas extraordinarios de atención a la diversidad en su último curso $-2^{\circ}$ de PDC y $2^{\circ}$ de PCPI-, con curricula diferentes al del aula ordinaria, es proporcionar unos 
conocimientos similares en una clase con menos alumnos y con una organización curricular diferente -definida en ámbitos-, o realmente lo que se pretende es separar los "malos alumnos" del resto, proporcionándoles un curriculum inferior con el que obtener el graduado en ESO de una manera mucho más sencilla.

\section{Análisis de datos}

Hemos establecido tres categorías de análisis.

- CANTIDAD: Equivale al número total de contenidos en comparación con los elementos del curriculum del programa tomado como referencia: $4^{\circ} \mathrm{ESO}$.

- RELEVANCIA: Depende del número de los contenidos, únicamente tomando los básicos, que existen en el PDC y el PCPI en comparación con el número de básicos que se encuentran en la referencia tomada $-4^{\circ}$ ESO-. Para determinar qué es básico y qué no lo es, como ya hemos señalado, se ha utilizado la opinión consensuada de los expertos, obtenida a través del método Delphi. Lo que hemos intentado es valorar la trascendencia de estos contenidos que no se encontraban en la legislación de alguno de los programas extraordinarios de atención a la diversidad, o incluso de los dos.

- EQUIVALENCIA CURRICULAR: Hemos intentado valorar en esta categoría de análisis los contenidos teniendo en cuenta tanto la cantidad de los mismos, así como su relevancia. Se trataría de comprobar con esta categoría si el curriculum de $2^{\circ}$ de PDC y $2^{\circ}$ de PCPI constituyen una herramienta de exclusión educativa o si por el contrario los contenidos legislados son equivalentes a los de $4^{\circ}$ de ESO.

Por último, a estas tres categorías les hemos dado distintos valores, con el objetivo de poder encasillar de la forma más precisa posible, todas las posibilidades que se daban en la presentación de los datos. En concreto, tales valores son los cinco siguientes:

- MUCHO MAYOR: Es muy superior a la referencia. Mayor del 50\%.

- MAYOR: Es superior a la referencia. Entre el $26 \%$ hasta el $50 \%$.

- SEMEJANTE: Es similar a la referencia. Puede ser tanto un poco superior a la referencia como un poco inferior a la misma. Entre el $25 \%$ inferior y el $25 \%$ superior.

- MENOR: Es inferior a la referencia. Entre el $26 \%$ y el $50 \%$.

- MUCHO MENOR: Es muy inferior a la referencia. Menor del 50\%.

\section{Resultados}

A continuación, vamos a exponer los resultados obtenidos en la investigación en cada una de las materias.

\section{Matemáticas}

Primero, en las siguientes dos tablas expondremos los resúmenes de los resultados encontrados y los explicaremos posteriormente. 


\begin{tabular}{|l|c|c|c|c|}
\hline \multicolumn{5}{|c|}{ Tabla 1. Resumen de contenidos de matemáticas por bloques } \\
\hline & C. BÁSICO & $4^{\circ}$ ESO & $2^{\circ}$ PDC & $2^{\circ}$ PCPI \\
\hline 1. Contenidos comunes & 4 & 6 & 6 & 5 \\
\hline 2. Números & 8 & 11 & 6 & 3 \\
\hline 3. Álgebra & 5 & 6 & 3 & 1 \\
\hline 4. Geometría & 3 & 3 & 2 & 2 \\
\hline 5. Funciones y Gráficos & 3 & 5 & 4 & $\varnothing$ \\
\hline 6. Estadística y Probabilidad & 3 & 8 & 3 & 1 \\
\hline
\end{tabular}

Tabla 2. Resumen de contenidos de matemáticas. Comparación con $4^{\circ}$ ESO

\begin{tabular}{|l|c|c|c|}
\hline CONTENIDOS & $\mathbf{4}^{\circ}$ ESO & $\mathbf{2}^{\circ}$ PDC & $\mathbf{2}^{\text {'PCPI }}$ \\
\hline Número total & 39 & 24 & 12 \\
\hline Básicos & 26 & 17 & 10 \\
\hline Excede Básicos & 13 & 7 & 2 \\
\hline CANTIDAD & REFERENCIA & MENOR & MUCHO MENOR \\
\hline RELEVANCIA & REFERENCIA & MENOR & MUCHO MENOR \\
\hline $\begin{array}{l}\text { EQUIVALENCIA } \\
\text { CURRICULAR }\end{array}$ & REFERENCIA & MENOR & MUCHO MENOR \\
\hline
\end{tabular}

Analizando en conjunto la cantidad de contenidos, podemos observar como de los 39 establecidos para $4^{\circ} \mathrm{ESO}$, menos de dos terceras partes -24-, están relacionados con contenidos de $2^{\circ}$ PDC, y en el PCPI ni siquiera una tercera parte de ellos $-12-$. Si el análisis lo realizamos de una manera más minuciosa, bloque a bloque, vemos que en el Programa de Diversificación Curricular existen enormes diferencias en Estadística y Probabilidad, y también en Álgebra, y son menores las mismas en el resto de bloques de contenidos. En el Programa de Cualificación Profesional Inicial, esta situación de falta de contenidos similares todavía se agrava más: el bloque de Funciones y Gráficos es inexistente, y en el de Estadística y Probabilidad hay un solo apartado testimonial. La excepción, la constituye el bloque tercero, el de Geometría, que podríamos considerarlo parecido al de $4^{\circ}$ de ESO: sólo hay uno de los tres contenidos que no está relacionado. Eso sí, en $2^{\circ}$ de PDC es idéntico el bloque de Contenidos Comunes, que es "el eje vertebrador de los conocimientos matemáticos que abarca". Este bloque hace referencia, entre otros, a un tema básico del curriculum: la resolución de problemas. También en $2^{\circ}$ de PCPI los contenidos en este bloque son muy similares abarcando casi la totalidad de los que se presentan en $4^{\circ}$ de ESO, 5 de los 6 .

Sobre todo en el PCPI, pero también en el PDC, los contenidos que no encuentran similitud con los de ESO son algunos muy relevantes -básicos-, como no podía ser de otra forma, debido a la gran diferencia en el número de los mismos. Contenidos como interés simple y compuesto, intervalos, operaciones con polinomios, identidades notables, análisis elemental de funciones y análisis elemental de la representatividad de las muestras estadísticas no se encuentran en ninguno de los dos programas. Pero, son todavía más esenciales las lagunas en el PCPI, donde contenidos como 
raíces y el teorema de Pitágoras tampoco aparecen. Por lo tanto, podemos afirmar que la equivalencia curricular de contenidos en Matemáticas no es similar en $4^{\circ}$ de ESO a la de $2^{\circ}$ de PDC, porque hay un importante número de contenidos que no aparecen, circunstancia ésta que se agrava considerablemente en el PCPI. En el $2^{\circ}$ de PDC la equivalencia curricular es menor y en el caso del PCPI mucho menor. De hecho, hay notables diferencias entre ambos programas extraordinarios: $2^{\circ} \mathrm{PCPI}$ presenta la mitad del número de contenidos que $2^{\circ}$ de PDC; además entre ellos hay una considerable distancia en la relevancia de los respectivos contenidos.

\section{Castellano}

Del mismo modo que hemos procedido en la materia de matemáticas, lo haremos en lengua castellana.

\begin{tabular}{|l|c|c|c|c|}
\hline \multicolumn{5}{|c|}{ Tabla 3. Resumen de contenidos de castellano por bloques } \\
\hline & C. BÁSICO & $4^{0}$ ESO & $2^{\text {O PDC }}$ & $2^{\circ}$ PCPI \\
\hline 1. Comunicación. Introducción & 1 & 2 & $\varnothing$ & 1 \\
\hline 1a. Estructuras formales de texto & 2 & 4 & 4 & $\varnothing$ \\
\hline 1b. Escuchar, hablar & 7 & 8 & 6 & 7 \\
\hline 1c. Leer & 2 & 4 & 4 & 2 \\
\hline 1d. Escribir & 3 & 4 & 3 & 3 \\
\hline 2. Lengua y Sociedad & 5 & 8 & $\varnothing$ & 3 \\
\hline 3a. Fonética y Ortografía & 1 & 3 & 1 & 1 \\
\hline 3b. Norma culta de la lengua & 2 & 5 & 1 & 2 \\
\hline 3c. Gramática & 1 & 5 & 3 & $\varnothing$ \\
\hline 3d. Léxico & 1 & 3 & $\varnothing$ & $\varnothing$ \\
\hline 4. Educación Literaria & 8 & 19 & 5 & 3 \\
\hline 5. Técnicas de Trabajo & 4 & 5 & 2 & 2 \\
\hline
\end{tabular}

Tabla 4. Resumen de contenidos de castellano. Comparación con $4^{\circ}$ ESO

\begin{tabular}{|l|c|c|c|}
\hline CONTENIDOS & $\mathbf{4}^{\circ}$ ESO & $\mathbf{2}^{\text {PPDC }}$ & $\mathbf{2}^{\text {OPCPI }}$ \\
\hline Número total & 70 & 31 & 27 \\
\hline Básicos & 37 & 18 & 21 \\
\hline Excede Básicos & 33 & 13 & 6 \\
\hline CANTIDAD & REFERENCIA & MUCHO MENOR & MUCHO MENOR \\
\hline RELEVANCIA & REFERENCIA & MUCHO MENOR & MENOR \\
\hline $\begin{array}{l}\text { EQUIVALENCIA } \\
\text { CURRICULAR }\end{array}$ & REFERENCIA & MUCHO MENOR & MENOR \\
\hline
\end{tabular}


Analizando la cantidad de contenidos de las tablas, nos damos cuenta de la gran diferencia entre $4^{\circ}$ ESO y los programas de PDC y PCPI. Mientras que nos encontramos con 70 contenidos para $4^{\circ} \mathrm{ESO}$, sólo se presentan 31 en el caso del Programa de Diversificación Curricular, y 27, todavía menos, en el Programa de Cualificación Profesional Inicial. Sí que es cierto que tenemos contenidos de ambos programas no relacionados con los de $4^{\circ} \mathrm{ESO}$, pero son los menos. Por lo tanto, los contenidos de $2^{\circ}$ PDC no están relacionados ni con la mitad de los de Educación Secundaria Obligatoria, pero la situación de los PCPI todavía es peor, al no hacerlo con casi las dos terceras partes de los mismos. Si analizamos la cantidad de contenidos de la asignatura de castellano con más detalle, observaremos que tenemos bloques enteros y apartados también íntegros sin ningún contenido. Esto ocurre en el PDC, en el bloque segundo, el denominado Lengua y Sociedad, y en el apartado 1, Introducción a la Comunicación, y en el 3d, Léxico. Hay otros apartados que sí tienen contenidos relacionados con $4^{\circ}$ de Educación Secundaria, pero presentan notables diferencias con el mismo: son los casos del apartado 3b, Norma Culta de la Lengua, que tan sólo tiene un contenido de cinco posibles; y el bloque cuarto, denominado Educación Literaria, con tan sólo cinco contenidos de diecinueve en $4^{\circ} \mathrm{ESO}$.

Es evidente que tal diferencia en el número de contenidos tiene que llevar consigo que al menos algunas de las partes ausentes tengan una enorme relevancia, son básicas. En el PDC, el bloque de Lengua y Sociedad, absolutamente sin contenidos, se refiere a la importancia de la lengua castellana en el mundo y su convivencia con el resto de lenguas del Estado, que no representa una cuestión menor, aunque apartados como el de Léxico, también sin contenidos, todavía puedan resultar de más importancia al tratarse de la riqueza en el vocabulario del alumno a la hora de escribir y de expresarse. En este mismo programa tenemos que fijarnos en aspectos absolutamente esenciales y básicos, y que apenas sí se consideran, como la Ortografía y la Educación Literaria. En el PCPI, podemos comentar prácticamente las mismas deficiencias, aunque puedan variar en algunos casos los apartados afectados. En este programa no se dedica ningún espacio a algo tan esencial como pueda ser la Gramática para una lengua, y se vuelve a caer en enormes lagunas de contenidos básicos que también se dan en PDC, como es el caso del Léxico y la Ortografía.

En consecuencia, la diferencia en los contenidos de castellano es abismal en ambos programas respecto a $4^{\circ}$ de Educación Secundaria, por lo que no podemos hablar de que sean equivalentes curricularmente. Hay un gran número de contenidos de los que se ha prescindido, y además, son extraordinariamente relevantes al parecer de los expertos, por lo que en ningún caso podemos hablar de exigencia parecida en los niveles que son objeto de nuestro estudio.

\section{Discusión}

Teniendo en cuenta los resultados obtenidos, podemos concluir que los contenidos de $2^{\circ}$ de PDC y de $2^{\circ}$ de PCPI, ni para matemáticas ni para castellano, son similares a los establecidos por el legislador para el aula ordinaria. Se establecen contenidos que tanto en número como en relevancia se encuentran muy lejanos a los que nos sirven de referencia $-4^{\circ}$ de ESO- e incluso a los que los expertos del Delphi han considerado como básicos. No cumple el legislador con su promesa de establecer curricula diferentes pero culturalmente semejantes.

Es cierto, que los aprendizajes del alumnado pueden ser distintos a los marcados en el curriculum oficial, pero no lo es menos que éste suele resultar fundamental en el devenir de lo que sucede en el aula. Difícilmente los alumnos y alumnas del PDC y del PCPI podían disfrutar del derecho a la educación desde una perspectiva más amplia 
que el del mero acceso a la escuela. No podían realmente alcanzar unos aprendizajes fundamentales y básicos para la vida al prescindir el legislador en el curriculum de contenidos esenciales. Sí que les resultaba más fácil conseguir el título de Graduado en ESO, pero principalmente por la rebaja sustancial que se producía en los contenidos de sus curricula.

Aunque estos dos programas -PDC y PCPI- se extinguieron a la finalización del curso 2015 , los resultados de la investigación son valiosos y pueden servir para la mejora de los curricula de futuras leyes educativas. Así pues, y entrando en el terreno propositivo, apostamos que para leyes venideras se elabore de forma consensuada un curriculum básico de las distintas materias, de manera que independientemente que el legislador apueste por un curriculum común para todo el alumnado o por curricula diferenciados para programas extraordinarios de atención a la diversidad, en todos ellos estén presentes los contenidos que nadie debe ignorar, porque todos los alumnos y alumnas, sin excepciones, tienen que formarse como ciudadanos y disfrutar del derecho a la educación.

Referencias

Bolívar, A. (2008). Ciudadanía y Competencias básicas. Sevilla: Editorial Fundación ECOEM.

Carbonell, J. (2014). Las pedagogías innovadoras y las visiones de los contenidos. Cuadernos de Pedagogía, 447, $42-45$.

Casanova, MA. (1995). Manual de evaluación educativa. Madrid: Editorial La Muralla.

Escudero, JM. (2006). Realidades y respuestas a la exclusión educativa. Cap. 6. En Juan Manuel Escudero y Juan Sáez (2006) Exclusión Social, Exclusión Educativa. Murcia: Editorial DM/ICE, Universidad de Murcia.

Escudero, JM. (2009). Fracaso escolar y exclusión educativa. Editorial Profesorado, Revista de Currículum y Formación de Profesorado, 13 (3), 3-9.

Gimeno, J. (1989). El curriculum: una reflexión sobre la práctica. Madrid: Editorial Morata.

Gimeno, J. (1998). Poderes inestables en educación. Madrid: Editorial Morata.

Gimeno, J. (2014). La sustantividad educativa de los contenidos. Cuadernos de Pedagogía 447, 14-17.

Landeta, J. (2002). El método Delphi. Una técnica de previsión del futuro. Barcelona: Editorial Ariel.

Pozo, JL. (2010). El aprendizaje de contenidos escolares y la adquisición de competencias. En Cesar Coll (2010) Desarrollo, aprendizaje y enseñanza en la educación secundaria. Barcelona: Editorial Graó.

Tomasevski, K. (2004). El asalto a la educación. Barcelona: Editorial Intermon Oxfam.

Torres, RM. (2006). Derecho a la educación es mucho más que acceso a la escuela. En Luis María Naya y Pauli Dávila (2006.). El derecho a la educación en un mundo globalizado. Tomo I, 43-58. Donostia: Editorial Espacio Universitario/ Erein. 